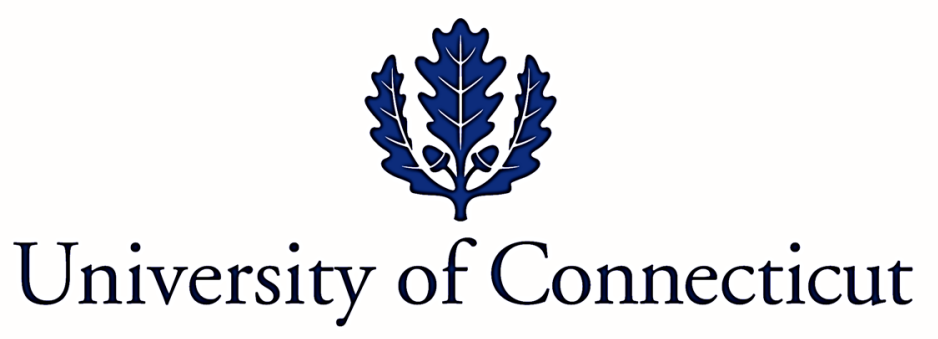

Department of Economics Working Paper Series

\title{
The Transformation Function, Technical
} Efficiency, and the CCR Ratio

by

Subhash C Ray

University of Connecticut

Working Paper 2017-09

June 2017

365 Fairfield Way, Unit 1063

Storrs, CT 06269-1063

Phone: (860) 486-3022

Fax: (860) 486-4463

http://www.econ.uconn.edu/

This working paper is indexed in RePEc, http://repec.org 


\title{
THE TRANSFORMATION FUNCTION, TECHNICAL EFFICIENCY, AND THE CCR RATIO
}

\author{
Subhash C Ray \\ Department of Economics \\ University of Connecticut \\ Storrs CT 06269-1063 \\ subhash.ray@uconn.edu
}

Charnes, Cooper, and Rhodes define the ratio of the virtual output to the virtual input as a measure of the technical efficiency of a multiple output multiple input firm. The aggregation weights used in constructing the virtual output and the virtual input may be arbitrarily chosen so long as the weights are non-negative and using these weights no firm's input-output bundle shows efficiency exceeding $100 \%$. In production economics, the ratio of aggregate output to aggregate input is a measure of total factor productivity and a direct link of the CCR ratio to technical efficiency is not obvious. Usually the CCR ratio is rationalized as efficiency by showing its equivalence to the Farrell efficiency measure. This paper offers a direct derivation of the CCR ratio measure of efficiency from a Transformation Function. We also show how the Banker, Charnes, Cooper (BCC) measure under variable returns to scale can be derived from the Transformation Function.

JEL Codes: D24; C60

Keywords: Supporting hyperplane; Convexity; Degree of Increasing Returns

The author is grateful to Rolf Färe and Rajiv Banker for valuable comments. The usual disclaimer applies. 


\section{THE TRANSFORMATION FUNCTION, TECHNICAL EFFICIENCY, AND THE CCR RATIO}

In production economics the technical efficiency of a decision making unit (generally described as a firm) producing a single output is measured by the ratio of the quantities of the actual output produced and the maximum producible output from the quantity of input it has used. In the multiple output case, it is the inverse of the maximum scalar by which its actual output bundle could be expanded without requiring any more input. Farrell's (1957) measure of technical efficiency (in the single output case) and Shephard's (1953) Distance Function (in the multiple output case) are examples of how technical efficiency is measured in economics. Charnes, Cooper, and Rhodes (CCR) (1978) introduced the maximum value of the ratio of a weighted sum of the observed output quantities to a weighted sum of input quantities used by a firm as a measure of its technical efficiency. Apart from non-negativity, the weights are constrained to be such that evaluated at these weights the ratio of the weighted output to the weighted input for any observed input-output bundle cannot exceed unity. In empirical applications, economists typically prefer the Shephard-Farrell approach mainly because they clearly link with the concept of a production possibility set. Analysts from OR and Management Science, on the other hand, usually work with the weights (also known as multipliers) and are not particularly concerned about their relation to underlying production technology. The link between the CCR ratio and the Shephard-Farrell efficiency measures is not obvious. In fact, as noted by Førsund (2013), as a ratio of aggregated output and aggregated input, the $\mathrm{CCR}$ ratio is more akin to total factor productivity. Of course, through an appropriate normalization, one can transform the CCR linear fractional functional programming problem into the radial Farrell technical efficiency measurement problem. However, such algebraic equivalence does not establish a conceptual equivalence between the two. It is true that CCR did include a single-output single-input example trying to show how their ratio measure reduces to what they call an 'engineering measure' of efficiency. Unfortunately, as discussed below, their example creates more confusion instead of providing a clarification.

The twofold objectives of this note are to show that (a) in the single output-single input case, the CCR ratio is a productivity index with the unit with the highest productivity treated as the base and (b)in the multiple-output multiple-input case, one can directly derive the CCR ratio as a measure of efficiency from a transformation function that is homogenous of degree 0 in outputs and inputs. This provides an interpretation of the vector of weights as the gradient of a tangent hyperplane to the production possibility set. We also show how the ratio form of the BCC measure under variable returns to scale can be derived from the transformation function. 


\section{The CCR Ratio and the 1-input 1-output Case}

Consider a sample of $N$ firms from some industry producing $m$ outputs from $n$ inputs. Suppose that $x^{j}=\left(x_{1 j}, x_{2 j}, \ldots x_{i j}, \ldots x_{n j}\right)$ is the input vector and $y^{j}=\left(y_{1 j}, y_{2 j}, \ldots, y_{r j}, \ldots y_{m j}\right)$ output vector of firm $j$ $(j=1,2, \ldots, N)$. The data set available to the analyst is the set of observed input-output bundles

$$
D=\left\{\left(x^{1}, y^{1}\right),\left(x^{2}, y^{2}\right), \ldots,\left(x^{N}, y^{N}\right)\right\}
$$

Now consider a firm with the input-output bundle $\left(x^{0}, y^{0}\right)$ that is an element of $D$. CCR define the efficiency of this firm as

$$
\begin{array}{ll}
h_{0}=\max & \frac{\sum_{r=1}^{m} u_{r} y_{r 0}}{\sum_{i=1}^{n} v_{i} x_{i 0}} \\
\text { s.t. } \quad & \frac{\sum_{r=1}^{m} u_{r} y_{r j}}{\sum_{i=1}^{n} v_{i} x_{i j}} \leq 1 ;(j=1,2, \ldots, N) \\
& u_{r}, v_{i} \geq 0 \quad(r=1,2, \ldots, m ; i=1,2, \ldots, n)
\end{array}
$$

Two things may be noted. First, as noted by Førsund $(2013,2017), h_{0}$ is essentially a measure of total factor productivity. The weights $u=\left(u_{1}, u_{2}, \ldots, u_{m}\right)$ and $v=\left(v_{1}, v_{2}, \ldots, v_{n}\right)$ are used to construct the aggregate output $Y_{0}=u^{\prime} y^{0}$ and aggregate input $X_{0}=v^{\prime} x^{0}$ for the firm under evaluation. Similarly, $Y_{j}=u^{\prime} y^{j}$ and $X_{j}=v^{\prime} x^{j}$ are the aggregate output and input for firm $j$. Thus, $h_{0}=\frac{Y_{0}}{X_{0}}$ is a measure of total factor productivity. It is well known that productivity and efficiency are closely related but different concepts. As noted by Ray (2004), efficiency is a normative measure while productivity is a descriptive 
measure of performance. Secondly, CCR provide little justification ${ }^{1}$ for the inequalities $\frac{Y_{j}}{X_{j}} \leq 1$ in their construction of $h_{0}$.

Obviously, CCR recognized that $h_{0}$ in (2) did not look like a ratio of actual and maximum producible outputs from a given quantity of input. That is why, in order to rationalize $h_{0}$ as a technical efficiency measure they gave the example of heat discharged from a given quantity of fuel and invoked what they called an engineering definition of efficiency. The actual heat discharged $y_{r}$ from a given quantity of fuel is compared with the maximum possible heat $y_{R}$ from the same quantity of fuel to obtain the efficiency measure

$$
E_{r}=\frac{y_{r}}{y_{R}} \in(0,1)
$$

CCR try to relate this to their ratio measure in (2). On page 431, they refer to three output quantities, $y_{r}$, $y_{R}$, and $y_{0}$. Here $y_{0}$ is the output of the unit under evaluation, $y_{r}$ is the output of any individual unit in the data set (could be the unit evaluated also) and $y_{R}$ is the maximum quantity of the output producible from the given input $x$ which is being used by all units. This is made explicit by their statement $x_{R}=x_{r}=x$. Next, they appeal to their constraints in (1) to conclude $u y_{R}=v x_{R}=v x_{0}$, and, therefore,

$$
h_{0}=\frac{u y_{0}}{v x_{0}}=\frac{u y_{0}}{u y_{R}}=\frac{y_{0}}{y_{R}}=E_{0} .
$$

However, even for this simple 1-input 1-output case, there are several interrelated questions. First, it is not clear from their writing whether $y_{R}$ is one of the observed output quantities. If not, in what sense is $h_{0}$ a measure of technical efficiency of the relevant unit? Second, why should the ratios in the constraints be bounded above at 1 ? Finally, in any realistic situation, the input quantities will differ across units. How does the maximum output producible depend on the quantity of input used?

\footnotetext{
${ }^{1}$ Of course if the vectors $u$ and $v$ were market prices, these constraints would mean that at these prices no unit could earn a positive profit. See in this context the discussion of 'shadow profit maximization' in Ray (2007) and Lelue (2013).
} 
Although CCR do not directly address these questions, it is possible to answer all of them by using a production function defining the maximum quantity of output producible from a specific quantity of input.

In the 1-output 1-input case, the problem in (2) becomes

$$
\begin{aligned}
& h_{0}=\max \frac{u y_{0}}{v x_{0}} \\
& \text { s.t. } \\
& \qquad \frac{u y_{j}}{v x_{j}} \leq 1 ;(j=1,2, \ldots, N) \\
& u, v \geq 0 .
\end{aligned}
$$

Now define $k=\frac{v}{u}$. Then (5) reduces to

$$
\begin{aligned}
& h_{0}=\max \frac{y_{0}}{k x_{0}} \\
& \text { s.t. } \\
& \qquad \frac{y_{j}}{k x_{j}} \leq 1 ;(j=1,2, \ldots, N) \\
& \quad k \geq 0 .
\end{aligned}
$$

Because $x_{0}$ and $y_{0}$ are given parameters, the problem in (6) is equivalent to the minimization problem

$$
\begin{aligned}
& h_{0}=\min k \\
& \text { s.t. } \\
& \qquad \frac{y_{j}}{x_{j}} \leq k ;(j=1,2, \ldots, N) \\
& \quad k \geq 0 .
\end{aligned}
$$

Clearly, the optimal value of $k$ in both (6) and (7) is

$$
\begin{gathered}
k^{*}=\max _{j}\left\{\frac{y_{j}}{x_{j}}\right\} \equiv\left(\frac{y}{x}\right)^{*} \\
h_{0}=\frac{\frac{y_{0}}{x_{0}}}{\max _{j}\left\{\frac{y_{j}}{x_{j}}\right\}}
\end{gathered}
$$


As described in Cooper, Dharmapala, and Thrall (1996), the CCR ratio is in essence a 'ratio of ratios'. Note, further, that this ratio of average productivities is really a productivity index.

At this point, define the production function

$$
f(x)=k x
$$

Then the objective function in (2) becomes $h_{0}=\frac{y_{0}}{f\left(x_{0}\right)}$ which is clearly a measure of efficiency. Further, the constraints are now $y_{j} \leq f\left(x_{j}\right),(j=1,2, \ldots, N)$ which follow from the definition of a production function. It is important to note that the production function defined in (10) exhibits constant returns to scale (CRS).

In most applications, it is quite unlikely that there will be a readily available scientific formula defining the maximum output $y_{R}$ producible from a specific input quantity $x$ and the analyst must rely on observed input-output data to estimate it as

$$
\hat{y}_{R}=k^{*} x=\max _{j}\left\{\frac{y_{j}}{x_{j}}\right\} x \text {. }
$$

Of course, when all firms use the same unit quantity, $x_{j}=x(j=1,2, \ldots, N)$ and in that special case,

$$
\hat{y}_{R}=\max _{j}\left\{y_{j}\right\} \text {. }
$$

But even when different units use different quantities of the input, the maximum producible output will be proportional to the quantity of input used and

$$
y_{j}^{*}=f\left(x_{j}\right)=k x_{j}
$$

and the technical efficiency of the unit producing output $y_{0}$ from input $x_{0}$ is

$$
\tau_{y}\left(x_{0}, y_{0}\right)=h_{0}=\frac{y_{0}}{f\left(x_{0}\right)}=\frac{\frac{y_{0}}{x_{0}}}{\max _{j}\left\{\frac{y_{j}}{x_{j}}\right\}}
$$

This simple explanation of the CCR ratio as a measure of output oriented technical efficiency breaks down in the multiple output multiple input case. As Førsund (2013) has pointed out there is hardly any scientific formula that spells out maximal combinations of multiple outputs that can be produced from bundles of multiple inputs. In addition, with multiple outputs, one cannot define a production function. 
However, one can still show that the CCR measure is a multiple output generalization of the Farrell measure of technical efficiency.

\section{From CCR back to Farrell: multiple outputs and multiple inputs}

As an alternative but equivalent formulation, of the problem in (2) consider the problem

$$
\psi=\frac{1}{h_{0}}=\min \frac{v^{\prime} x^{0}}{u^{\prime} y^{0}} \text { s.t. } \frac{v^{\prime} x^{j}}{u^{\prime} y^{j}} \geq 1,(j=1,2, \ldots, N) ; u, v \geq 0 .
$$

In light of the fact that both the objective function and the constraints are homogeneous of degree zero in $u$ and $v$, one can use the normalization

$$
u^{\prime} y^{0}=1
$$

The optimization problem in (15) would then become

$$
\begin{aligned}
& \psi=\min \sum_{i=1}^{n} v_{i} x_{i 0} \\
\text { s.t. } & \sum_{i=1}^{n} v_{i} x_{i j}-\sum_{r=1}^{m} u_{r} y_{r j} \geq 0,(j=1,2, \ldots, N) \\
& \sum_{r=1}^{m} u_{r} y_{r 0}=1 ; \quad u_{r}, v_{i} \geq 0 ;(r=1,2, \ldots, m ; i=1,2, \ldots, n) .
\end{aligned}
$$

An intuitive interpretation of problem (17) is helpful. At the chosen vector of output prices, the observed output bundle of the unit under evaluation constitutes one unit of the composite output

$$
Y_{0} \equiv u^{\prime} y^{0}=1
$$

As defined earlier, $X_{0}$ is a measure of the aggregate input of the firm under evaluation while $\left(X_{0}^{j}, Y_{0}^{j}\right)$ is the aggregated input-output bundle of firm $j$ evaluated at the prices $u$ for the outputs and $v$ for the inputs. Because, $Y_{0}$ equals unity (by normalization) the objective function is the measured input-output ratio or the inverse of the average productivity of the firm under investigation. An implication of the constraints is that no firm can have an input-output ratio less than (equivalently productivity greater than) unity. 
It needs to be emphasized that both the output price vector $u=\left(u_{1}, u_{2}, \ldots, u_{m}\right)$ and the input price vector $v=\left(v_{1}, v_{2}, \ldots, v_{n}\right)$ have been selected specifically for the firm under evaluation. When some other firm (say firm $q$ instead of firm $k$ ) is being evaluated, a different set of input and output prices will be selected. The fact the optimal weights given to inputs or outputs while measuring efficiency vary across firms is one reason why they are (mistakenly) considered to be arbitrary. We will return to this issue presently. The maximization problem dual to the minimization problem in (17) is

$$
\begin{gathered}
\max \varphi \\
\text { s.t. } \sum_{j=1}^{N} \lambda_{j} x_{i j} \leq x_{i 0},(i=1,2, \ldots, n) ; \\
\sum_{j=1}^{N} \lambda_{j} y_{r j} \geq \varphi y_{r 0},(r=1,2, \ldots, m) ; \\
\lambda_{j} \geq 0,(j=1,2, \ldots, N) ; \varphi \text { unrestricted. }
\end{gathered}
$$

The optimal solution of (19), $\varphi^{*}$, defines the benchmark output bundle $y^{*}=\varphi^{*} y^{0}$ against which the actual output, $y^{0}$, should be compared. By standard duality results, $\varphi^{*}=\psi^{*}$ and $h_{0}=\frac{1}{\psi^{*}}$. This shows the equivalence between the CCR ratio measure and the Farrell measure of technical efficiency.

\section{From Production Economics to the CCR Ratio}

The algebraic equivalence between the CCR and the Farrell measures by itself does not provide any economic content to the CCR ratio. We now show how one can derive the CCR measure from the concept of efficiency in neoclassical production economics.

The foundation of neoclassical theory of production is the production possibility set:

$$
T=\{(x, y): y \text { can be produced from } x\} \text {. }
$$

An input-output bundle $(x, y)$ constitutes a feasible production plan when $y$ can be produced from $x$. Thus, every feasible production plan is an element of the production possibility set, $T$. Stated differently, an input-output bundle that does not lie in $T$ is not feasible. We make the following assumptions about $T$ :

A1. Inputs are freely disposable. Thus, if $\left(x^{0}, y^{0}\right) \in T$ and $x^{1} \geq x^{0}$, then $\left(x^{1}, y^{0}\right) \in T$.

A2. Outputs are freely disposable. Thus, if $\left(x^{0}, y^{0}\right) \in T$ and $y^{1} \leq y^{0}$, then $\left(x^{0}, y^{1}\right) \in T$.

A3. The production possibility set is convex. 
Often the production possibility set is defined as

$$
T=\{(x, y): F(x, y) \leq 0\} .
$$

The function $F(x, y)=0$ is the implicit production function in the single output case and the transformation function in the multiple output case. Assuming that the function is differentiable, free disposability of inputs and outputs imply $\frac{\partial F}{\partial x_{i}} \equiv F_{i} \leq 0$ for every input $i$ and $\frac{\partial F}{\partial y_{r}} \equiv F_{r} \geq 0$ for every output $r$. It can be seen that if $F\left(x^{0}, y^{0}\right)>0,\left(x^{0}, y^{0}\right)$ is infeasible. The set

$$
G=\{(x, y): F(x, y)=0\}
$$

is known as the graph of the technology. Every $(x, y) \in G$ is technically efficient. If one assumes that the technology exhibits constant returns to scale globally, $(x, y) \in T \Rightarrow(k x, k y) \in T$ for all $k \geq 0$. In that case

$$
F(x, y)=F(k x, k y)
$$

That is, the transformation function is homogeneous of degree 0 in $x$ and $y$.

We may now define efficiency relative to the graph of the technology. Consider some feasible inputoutput bundle $\left(x^{0}, y^{0}\right)$. Assume that $F\left(x^{0}, y^{0}\right)<0$ so that $\left(x^{0}, y^{0}\right)$ is an inefficient bundle. Next consider the Shephard Distance Function

$$
D\left(x^{0}, y^{0}\right)=\min \beta:\left(x^{0}, \frac{1}{\beta} y^{0}\right) \in T \Leftrightarrow F\left(x^{0}, \frac{1}{\beta} y^{0}\right)=0 .
$$

It is the inverse of the largest scalar $\delta$ such that $F\left(x^{0}, \delta y^{0}\right)=0$ and is the same as the output oriented Farrell efficiency. Clearly, for $\left(x^{0}, y^{0}\right), \delta>1$ and $\beta<1$.

Focus now on the efficient input-output bundle $\left(x^{0}, y_{0}^{*}\right)=\left(x^{0}, \delta y^{0}\right)$ lying on the graph of the technology. Thus $F\left(x^{0}, y_{0}^{*}\right)=0$. Now, due to homogeneity of degree 0 ,

$$
F\left(x^{0}, y_{0}^{*}\right)=\sum_{i}\left(\frac{\partial F}{\partial x_{i}}\right)_{x^{0}, y_{0}^{*}} x_{i 0}+\sum_{r}\left(\frac{\partial F}{\partial y_{r}}\right)_{x^{0}, y_{0}^{*}} y_{r 0}^{*}=0
$$

Define

$$
F_{i}^{0} \equiv\left(\frac{\partial F}{\partial x_{i}}\right)_{x^{0}, y_{0}^{*}}(i=1,2, \ldots, n)
$$

and

$$
F_{r}^{0} \equiv\left(\frac{\partial F}{\partial y_{r}}\right)_{x^{0}, y_{0}^{*}}(r=1,2, \ldots, m) .
$$

Then (25) becomes 


$$
\sum_{i} F_{i}^{0} x_{i 0}+\sum_{r} F_{r}^{0} y_{r 0}^{*}=0
$$

Because $y_{r 0}^{*}=\delta y_{r 0},(28)$ leads to

$$
\delta \sum_{r} F_{r}^{0} y_{r 0}=-\sum_{i} F_{i}^{0} x_{i 0}
$$

Thus,

$$
\beta=\frac{1}{\delta}=\frac{\sum_{r} F_{r}^{0} y_{r 0}}{-\sum_{i} F_{i}^{0} x_{i 0}} .
$$

Finally, define $u_{r} \equiv F_{r}^{0}$ and $v_{i} \equiv-F_{i}^{0}$ to derive

$$
\beta=\frac{1}{\delta}=\frac{\sum_{r} u_{r} y_{r 0}}{\sum_{i} v_{i} x_{i 0}} .
$$

This shows that the CCR ratio in (2) is the same as the Shephard Distance Function or the Farrell measure of technical efficiency.

One still needs a rationale for the inequality constraints in the CCR optimization problem. For this, we may appeal to a well-known theorem from linear algebra about a supporting hyperplane to a convex set.

Theorem: If $A=\{w: f(w) \leq k\}$ is a closed and convex set, the partial derivatives $\frac{\partial f}{\partial w_{i}}$ are continuous, $w^{0} \in A$ is a boundary point satisfying $f\left(w^{0}\right)=k$, and not all of the partial derviatives evaluated at $w^{0}$ are zero, then the tangent hyperplane through $w^{0}, p(w)=f\left(w^{0}\right)+\Delta f_{\left(w^{0}\right)}{ }^{\prime}\left(w-w^{0}\right)$, is a supporting hyperplane to $A$ such that $p\left(w^{0}\right)=f\left(w^{0}\right)$ and $p(w) \leq k \forall w \in A$.

Proof: See Nikaido (1970) pp 199-200. ${ }^{2}$

In the present context, $T=\{(x, y): F(x, y) \leq 0\}$ is closed and convex by assumption. Also, at the inputoutput bundle $\left(x^{0}, y_{0}^{*}\right), F\left(x^{0}, y_{0}^{*}\right)=0$. Thus, the supporting hyperplane at this point is

\footnotetext{
${ }^{2}$ In this paper, as in Nikaido (1970), we assume that the Production Correspondence is differentiable. Nikaido also outlines a proof for the more general case.
} 


$$
\begin{aligned}
& p(x, y)=\left[F\left(x^{0}, y_{*}^{0}\right)-\sum_{i}\left(\frac{\partial F}{\partial x_{i}}\right)_{x^{0}, y_{0}^{*}} x_{i 0}-\sum_{r}\left(\frac{\partial F}{\partial y_{r}}\right)_{x^{0}, y_{0}^{*}} y_{r 0}^{*}\right]+\sum_{i}\left(\frac{\partial F}{\partial x_{i}}\right)_{x^{0}, y_{0}^{*}} x_{i}+\sum_{r}\left(\frac{\partial F}{\partial y_{r}}\right)_{x^{0}, y_{0}^{*}} y_{r} \\
& =\sum_{i}\left(\frac{\partial F}{\partial x_{i}}\right)_{x^{0}, y_{0}^{*}} x_{i}+\sum_{r}\left(\frac{\partial F}{\partial y_{r}}\right)_{x^{0}, y_{0}^{*}} y_{r} .
\end{aligned}
$$

Note that the expression inside the square brackets equals 0 due to homogeneity of degree 0 (as shown in (25) above).

Moreover, $p\left(x^{0}, y_{0}^{*}\right)=F\left(x^{0}, y_{0}^{*}\right)=0$ Hence, using the earlier definitions $u_{r}=F_{r}^{0}$ and $v_{i}=-F_{i}^{0}$, the supporting (or tangent) hyperplane is

$$
p(x, y)=\sum_{r} u_{r} y_{r}-\sum_{i} v_{i} x_{i}=0
$$

Finally, for every $\left(x^{j}, y^{j}\right) \in D, F\left(x^{j}, y^{j}\right) \leq 0$. Hence, by virtue of the theorem above,

$$
\sum_{r} u_{r} y_{r j}-\sum_{r} v_{i} x_{i j} \leq 0
$$

This leads to the constraints

$$
\frac{\sum_{r} u_{r} y_{r j}}{\sum_{r} v_{i} x_{i j}} \leq 1 ;(j=1,2, \ldots, N) .
$$

\section{Variable Returns to Scale and the Ratio form of the BCC Measure}

Banker, Charnes, and Cooper (BCC) (1984) relaxed the constant returns to scale assumption to allow locally increasing, constant, and diminishing returns to scale at different points on different segments of the production frontier. For this, they appended the constraint that the non-negative $\lambda$-weights should add up to unity in the optimization problem in (20) above. The output-oriented BCC problem is

$$
\begin{gathered}
\max \varphi \\
\text { s.t. } \sum_{j=1}^{N} \lambda_{j} x_{i j} \leq x_{i 0},(i=1,2, \ldots, n) \\
\sum_{j=1}^{N} \lambda_{j} y_{r j} \geq \varphi y_{r 0},(r=1,2, \ldots, m) ; \\
\sum_{j=1}^{N} \lambda_{j}=1 \\
\lambda_{j} \geq 0,(j=1,2, \ldots, N) ; \varphi \text { unrestricted. }
\end{gathered}
$$


The dual of this maximization problem is

$$
\begin{aligned}
& \min v_{0}+\sum_{i=1}^{n} v_{i} x_{i 0} \\
& \text { s.t. } v_{0}+\sum_{i=1}^{n} v_{i} x_{i j}-\sum_{r=1}^{m} u_{r} y_{r j} \geq 0,(j=1,2, \ldots, N) ; \\
& \sum_{r=1}^{m} u_{r} y_{r 0}=1 ; u_{r}, v_{i} \geq 0 ;(r=1,2, \ldots, m ; i=1,2, \ldots, n) ; v_{0} \text { unrestricted. }
\end{aligned}
$$

This is equivalent to maximizing

$$
\gamma=\frac{1}{v_{0}+\sum_{i=1}^{n} v_{i} x_{i 0}}
$$

Further, using (38) and the constraint $\sum_{r=1}^{m} u_{r} y_{r 0}=1$, the problem in (37) can be equivalently formulated as

$$
\begin{array}{ll}
\gamma=\max & \frac{\sum_{r=1}^{m} u_{r} y_{r 0}}{v_{0}+\sum_{i=1}^{n} v_{i} x_{i 0}} \\
& \\
& \quad \frac{\sum_{r=1}^{m} u_{r} y_{r j}}{v_{0}+\sum_{i=1}^{n} v_{i} x_{i j}} \leq 1, \quad(j=1,2, \ldots, N) ; \\
& u_{r}, v_{i} \geq 0 ;(r=1,2, \ldots, m ; i=1,2, \ldots, n) ; v_{0} \text { unrestricted. }
\end{array}
$$

This is the ratio form of the output-oriented BCC model.

We now show how this model can be derived directly from the Transformation function allowing variable returns to scale. As before, we start from the inefficient bundle $\left(x^{0}, y^{0}\right)$ which is radially projected to the efficient bundle $\left(x^{0}, y_{0}^{*}\right)$ where $y_{0}^{*}=t y^{0}$. This time, however, the tangent hyperplane through $\left(x^{0}, y_{0}^{*}\right)$ is

$$
\begin{aligned}
& p(x, y)=\left[F\left(x^{0}, y_{*}^{0}\right)-\sum_{i}\left(\frac{\partial F}{\partial x_{i}}\right)_{x^{0}, y_{0}^{*}} x_{i 0}-\sum_{r}\left(\frac{\partial F}{\partial y_{r}}\right)_{x^{0}, y_{0}^{*}} y_{r 0}^{*}\right]+\sum_{i}\left(\frac{\partial F}{\partial x_{i}}\right)_{x^{0}, y_{0}^{*}} x_{i}+\sum_{r}\left(\frac{\partial F}{\partial y_{r}}\right)_{x^{0}, y_{0}^{*}} y_{r} \\
& =\left[-\sum_{i}\left(\frac{\partial F}{\partial x_{i}}\right)_{x^{0}, y_{0}^{*}} x_{i 0}-\sum_{r}\left(\frac{\partial F}{\partial y_{r}}\right)_{x^{0}, y_{0}^{*}} y_{r 0}^{*}\right]+\sum_{i}\left(\frac{\partial F}{\partial x_{i}}\right)_{x^{0}, y_{0}^{*}} x_{i}+\sum_{r}\left(\frac{\partial F}{\partial y_{r}}\right)_{x^{0}, y_{0}^{*}} y_{r} \\
& \equiv \kappa_{0}+\sum_{i}\left(\frac{\partial F}{\partial x_{i}}\right)_{x^{0}, y_{0}^{*}} x_{i}+\sum_{r}\left(\frac{\partial F}{\partial y_{r}}\right)_{x^{0}, y_{0}^{*}} y_{r}
\end{aligned}
$$


Note that $p\left(x^{0}, y_{0}^{*}\right)=F\left(x^{0}, y_{0}^{*}\right)=0$. That is,

$$
\begin{aligned}
& \kappa_{0}+\sum_{i}\left(\frac{\partial F}{\partial x_{i}}\right)_{x^{0}, y_{0}^{*}} x_{i 0}+\sum_{r}\left(\frac{\partial F}{\partial y_{r}}\right)_{x^{0}, y_{0}^{*}} y_{r 0}^{*} \\
& =\kappa_{0}+\sum_{i}\left(\frac{\partial F}{\partial x_{i}}\right)_{x^{0}, y_{0}^{*}} x_{i 0}+t \sum_{r}\left(\frac{\partial F}{\partial y_{r}}\right)_{x^{0}, y_{0}^{*}} y_{r 0} \\
& =0
\end{aligned}
$$

Hence,

$$
\frac{1}{t}=\frac{\sum_{r}\left(\frac{\partial F}{\partial y_{r}}\right)_{x^{0}, y_{0}^{*}} y_{r 0}}{-\kappa_{0}-\sum_{i}\left(\frac{\partial F}{\partial x_{i}}\right)_{x^{0}, y_{0}^{*}} x_{i 0}}
$$

Define $v_{0}=-\kappa_{0}$. Then, using the old definitions of $u_{r}$ and $v_{i}$

$$
\frac{1}{t}=\frac{\sum_{r} u_{r} y_{r 0}}{v_{0}+\sum_{i} v_{i} x_{i 0}}
$$

The tangent hyperplane is

$$
p(x, y)=\kappa_{0}-\sum_{i} v_{i} x_{i 0}+\sum_{r} u_{r} y_{r 0}=0
$$

and by convexity of the production possibility set,

$$
\begin{aligned}
& \kappa_{0}-\sum_{i} v_{i} x_{i j}+\sum_{r} u_{r} y_{r j} \leq 0 \\
& \Rightarrow \sum_{r} u_{r} y_{r j} \leq-\kappa_{0}+\sum_{i} v_{i} x_{i j}=v_{0}+\sum_{i} v_{i} x_{i j} \\
& \Rightarrow \frac{\sum_{r} u_{r} y_{r j}}{v_{0}+\sum_{i} v_{i} x_{i j}} \leq 1 ;(j=1,2, \ldots, N) .
\end{aligned}
$$

To relate the sign of the intercept $\kappa_{0}$ (and, hence, of $v_{0}=-\kappa_{0}$ ) to (local) returns to scale properties of the technology, we refer to the concept of the degree of increasing returns defined by Starrett (1977). For an infinitesimally small movement along the frontier $F(x, y)=0$ from the initial point $\left(x^{0}, y_{0}^{*}\right)$, 


$$
\begin{aligned}
& d F=\sum_{i}\left(\frac{\partial F}{\partial x_{i}}\right)_{x^{0}, y_{0}^{*}} d x_{i}+\sum_{r}\left(\frac{\partial F}{\partial y_{r}}\right)_{x^{0}, y_{0}^{*}} d y_{r}=0 \\
& \Rightarrow \sum_{i}\left(\frac{\partial F}{\partial x_{i}}\right)_{x^{0}, y_{0}^{*}} x_{i 0} \frac{d x_{i}}{x_{i o}}+\sum_{r}\left(\frac{\partial F}{\partial y_{r}}\right)_{x^{0}, y_{0}^{*}} y_{r 0}^{*} \frac{d y_{r}}{y_{r 0}^{*}}=0
\end{aligned}
$$

Now suppose, $\frac{d x_{i}}{x_{i 0}}=q_{1}$ for every input $i$ and $\frac{d y_{r}}{y_{r 0}^{*}}=q_{2}$ for each output $r$. Then (46) implies

$$
q_{1} \sum_{i}\left(\frac{\partial F}{\partial x_{i}}\right)_{x^{0}, y_{0}^{*}} x_{i 0}+q_{2} \sum_{r}\left(\frac{\partial F}{\partial y_{r}}\right)_{x^{0}, y_{0}^{*}} y_{r 0}^{*}=0
$$

That is

$$
\frac{q_{2}}{q_{1}}=\frac{-\sum_{i}\left(\frac{\partial F}{\partial x_{i}}\right)_{x^{0}, y_{0}^{*}} x_{i 0}}{\sum_{r}\left(\frac{\partial F}{\partial y_{r}}\right)_{x^{0}, y_{0}^{*}} y_{r 0}^{*}} .
$$

If $\frac{q_{2}}{q_{1}}=1$, a given proportionate change in all inputs results in the same proportionate change in all outputs. This corresponds to locally constant returns to scale. Locally increasing returns holds if $\frac{q_{2}}{q_{1}}>1$. Similarly, $\frac{q_{2}}{q_{1}}<1$ implies locally diminishing returns to scale.

Starrett (1977) defined the degree of increasing returns as

$$
D I R=\frac{q_{2}}{q_{1}}-1
$$

In the present context, the DIR at $\left(x^{0}, y_{0}^{*}\right)$ is

$$
\begin{gathered}
D I R=\frac{q_{2}}{q_{1}}-1=\frac{-\sum_{i}\left(\frac{\partial F}{\partial x_{i}}\right)_{x^{0}, y_{0}^{*}} x_{i 0}-\sum_{r}\left(\frac{\partial F}{\partial y_{r}}\right)_{x^{0}, y_{0}^{*}} y_{r 0}^{*}}{\sum_{r}\left(\frac{\partial F}{\partial y_{r}}\right)_{x^{0}, y_{0}^{*}} y_{r 0}^{*}} \\
=\frac{\kappa_{0}}{\sum_{r}\left(\frac{\partial F}{\partial y_{r}}\right)_{x^{0}, y_{0}^{*}} y_{r 0}^{*}} .
\end{gathered}
$$

Note that the denominator in (50) is strictly positive. Hence, the sign of DIR is the same as the sign of the numerator ${ }^{3}$.

\footnotetext{
${ }^{3}$ Banker and Thrall (1992) show that the intercept is negative, positive, or zero when the input-output bundle (if technically efficient) or its efficient projection (if inefficient) is, respectively, smaller than, larger than, or equal to its
} 
We now have three possible cases:

(a) Locally increasing returns to scale. DIR $>0 \Leftrightarrow \frac{q_{2}}{q_{1}}>1 \Rightarrow \kappa_{0}>0 \Rightarrow v_{0}<0$.

(b) Locally constant returns to scale. DIR $=0 \Leftrightarrow \frac{q_{2}}{q_{1}}=1 \Rightarrow \kappa_{0}=0 \Rightarrow v_{0}=0$.

(c) Locally diminishing returns to scale. DIR $<0 \Leftrightarrow \frac{q_{2}}{q_{1}}<1 \Rightarrow \kappa_{0}<0 \Rightarrow v_{0}>0$.

\section{About Weight Restrictions}

The paper ends with a note of caution about imposition of weight restrictions in the multiplier for of the DEA problem. As we have seen above, the multipliers (or the aggregation weights) correspond to the gradient of the supporting hyperplane to the graph at the efficient projection of an inefficient input-output bundle. Imposition of weight restrictions amounts to arbitrarily constraining the marginal rates of substitution between pairs of inputs or marginal rates of transformation between pairs of outputs. Practitioners often complain that these 'implicit' price ratios are arbitrary because they can diverge significantly from actual ratios of market prices. Indeed, these relative weights are measures of opportunity costs determined by the estimated technology and can differ significantly from market prices which are determined by the interaction between demand and supply. It is only in the long run equilibrium of a competitive production-and-exchange economy that the consumer's marginal rates of substitution between two goods and the producer's marginal rate of transformation between the same pair of goods will both be equal to the ratio of market prices.

\section{Summary}

This paper shows how the CCR ratio measure of efficiency along with the associated inequality constraints can be derived from a conventional Production Correspondence defining the multiple output multiple input technology under globally constant returns to scale. We also provide a similar derivation of that the BCC measure of output-oriented efficiency under variable returns to scale. In particular, we show how the sign of the constant intercept is determined by the nature of local returns to scale.

\section{References}

Banker, R.D., A. Charnes, and W.W. Cooper (1984), "Some Models for Estimating Technical and Scale

most productive scale size (MPSS). Hence, locally increasing, dimishing, or constant returns to scale holds at that point. 
Inefficiencies in Data Envelopment Analysis," Management Science, 30:9 (September), 1078-92.

Banker, R.D. and R.M. Thrall (1992) "Estimation of Returns to Scale using Data Envelopment Analysis"; European Journal of Operational Research; 62, 74-84.

Charnes, A., W.W. Cooper, and E. Rhodes (1978) "Measuring the Efficiency of Decision Making Units," European Journal of Operational Research 2:6 (November), 429-44.

Cooper, W.W., R.G. Thompson, and R.M. Thrall. 1996. Introduction: Extensions and new developments in DEA, Annals of Operations Research, 66, 3-45.

Førsund, F.R.. (2013) Weight restrictions in DEA: misplaced emphasis? J Prod Anal 40:271-283

Førsund, F.R.. (2017) Economic Interpretation of DEA; Socio-Economic Planning Sciences (forthcoming)

Leleu, H. (2013) Inner and outer approximations of technology: A shadow profit approach; Omega 41 $868-871$

Nikaido, H. (1970) Introduction to Sets and mapping in Modern Economics (North Holland)

Ray, S.C. (2004) Data Envelopment Analysis: Theory and Techniques for Economics and Operations Research (Cambridge)

Ray, S.C. (2007) Shadow profit maximization and a measure of overall inefficiency; J Prod Anal $27: 231-236$

Shephard,R.W. (1953) Cost and production Functions (Princeton)

Starrett, D.A. (1977) "Measuring Returns to Scale in the Aggregate, and the Scale Effect of Public Goods"; Econometrica , 45:6, 1439-1455. 